LA W RENCE LIVERMORE NATIONAL LABORATORY

\section{High Resolution K Shell X-Ray Spectroscopy Experiments}

J. Seely, U. Feldman, L. Marlin

December 19, 2012 
This document was prepared as an account of work sponsored by an agency of the United States government. Neither the United States government nor Lawrence Livermore National Security, LLC, nor any of their employees makes any warranty, expressed or implied, or assumes any legal liability or responsibility for the accuracy, completeness, or usefulness of any information, apparatus, product, or process disclosed, or represents that its use would not infringe privately owned rights. Reference herein to any specific commercial product, process, or service by trade name, trademark, manufacturer, or otherwise does not necessarily constitute or imply its endorsement, recommendation, or favoring by the United States government or Lawrence Livermore National Security, LLC. The views and opinions of authors expressed herein do not necessarily state or reflect those of the United States government or Lawrence Livermore National Security, LLC, and shall not be used for advertising or product endorsement purposes.

This work performed under the auspices of the U.S. Department of Energy by Lawrence Livermore National Laboratory under Contract DE-AC52-07NA27344. 


\title{
High Resolution K Shell X-Ray Spectroscopy Experiments
}

\author{
Final Report for NIF Concept Development, 30 March 2012 \\ John Seely, Uri Feldman, and Layne Marlin, Artep Inc. \\ LLNL Subcontract NO. B598517
}

\section{Subcontractor:}

Artep Inc.

Ronen Feldman

2922 Excelsior Springs Court, Ellicott City, MD 21042

Phone: (410) 480-2700
LLNS Contract Administrator:

Angela Merritt

Phone: (925) 422-5721

Fax: $\quad$ (925) 422-9294

E-Mail: merritt23@1lnl.gov

\section{LLNS Technical Representative: Don Correll}

\section{$\underline{\text { Statement of Work }}$}

The Subcontractor is to design a spectrometer that can be fielded in a NIF DIM that has three cylindrically-bent transmission crystals covering (1) 6-20 keV, (2) $10-150 \mathrm{keV}$, and (3) $50-511 \mathrm{keV}$. This work will build on the NRL Dual Crystal Spectrometer (DCS) having two crystals covering 10-50 keV and 20-120 keV and fielded at the LLNL/Titan and LLE/Omega laser facilities.

The new work will demonstrate the feasibility of:

(a) Extending coverage down to $6 \mathrm{keV}$, which involves thinning the crystal from the nominal 200-250 micron to 100 micron and bending the thin crystal on a form with ROC in the $10-25 \mathrm{~cm}$ range, and

(b) Extending the coverage up to $511 \mathrm{keV}$ by utilizing a thick crystal or combination of crystals (at least $1 \mathrm{~mm}$ thick) and bending onto a form with ROC in the 1-2 $\mathrm{m}$ range.

The experimental research will include bending a $1 \mathrm{~mm}$ thick gold single crystal onto a cylindrical lens form and tested using the NRL Cs-137 $662 \mathrm{keV}$ source. The project will experimentally demonstrate the feasibility of tasks (a) and (b), and based on this feasibility the mechanical and optical design of a threecrystal spectrometer with image plate detection and covering the $6-511 \mathrm{keV}$ range will be carried out and provided to LLNL for consideration as a NIF DIM instrument.

The deliverables will include a final report detailing the experimental crystal development and the CAD design of the DIM-compatible spectrometer. 


\section{Distribution of the Final Report:}

Reports shall be separately addressed and transmitted to:

Lawrence Livermore National Laboratory

Attention: (Intended Recipient, see below)

P.O. Box 808

7000 East Avenue

Livermore, CA 94551

Type Report $\quad$ No. of Copies $\quad \underline{\text { Recipient }}$

Final 11 Don Correll L-637, correll1@1lnl.gov

Final 1 Angela Merritt L-443, merritt23@1lnl.gov

A preliminary report of the work on this project was presented at the NIF Users Workshop at LLNL during 13-15 February 20012 which was attended by John Seely and Uri Feldman.

\section{Spectrometer Design}

\section{a) Introduction}

The instrument consists of three spectrometer channels and is named TRI-SPEC. Each spectrometer channel has a cylindrically bent transmission crystal, and as discussed below the three ranges of energy coverage are (1) $6-20 \mathrm{keV}$, (2) $18-120 \mathrm{keV}$, and (3) $50-300 \mathrm{keV}$ (600 keV in the second diffraction order). The instrument is designed to be deployed in a DIM. The spectrometer design is based on the NRL Dual Crystal Spectrometer (DCS) having two crystals covering 10-50 keV and 20-120 keV and that was successfully fielded at the LLNL/Titan laser facility and at the LLE/Omega laser facility in a TIM.

The spectrometer is designed with mechanisms that allow target to crystal standoff distances of $600 \mathrm{~mm}$ or $1000 \mathrm{~mm}$. This allows flexibility for a smaller or larger standoff depending on the type of target and the exclusion zones near the target.

Shown in Fig. 1 is the three-channel instrument deployed in a DIM with $600 \mathrm{~mm}$ target to crystal standoff distance. Illustrated is a laser pointing system that utilizes in-vacuum lasers as used on previous LLNL diagnostics. An alternative pointing system could be implemented as determined by LLNL.

The alignment lasers and the critical drive mechanisms are mounted behind blast shields. The blue set of lasers (one set above and one below the mid-plane of the instrument) is used to align the spectrometers at the $600 \mathrm{~mm}$ distance. The lower or red pairs of lasers are used to align the diagnostic at the $1000 \mathrm{~mm}$ distance. 
Due to the availability of space within the DIM confines, the three spectrometers are mounted next to each other with the spectral dispersion along the vertical direction and the crossover slit openings horizontal. As seen in Fig. 1, the highest-energy and longest spectrometer channel \#3 is located in the center of the instrument and functions at the rigid spine of the instrument for attaching to the DIM. The two lower-energy channels \#1 and \#2 are positioned on either side of the channel \#3 and can be tipped outward to accommodate the larger $1000 \mathrm{~mm}$ standoff distance using novel drive mechanisms that are described below. Each of the three channels has an image plate (IP) detector assembly allowing the IP to be conveniently removed from the top of the instrument.

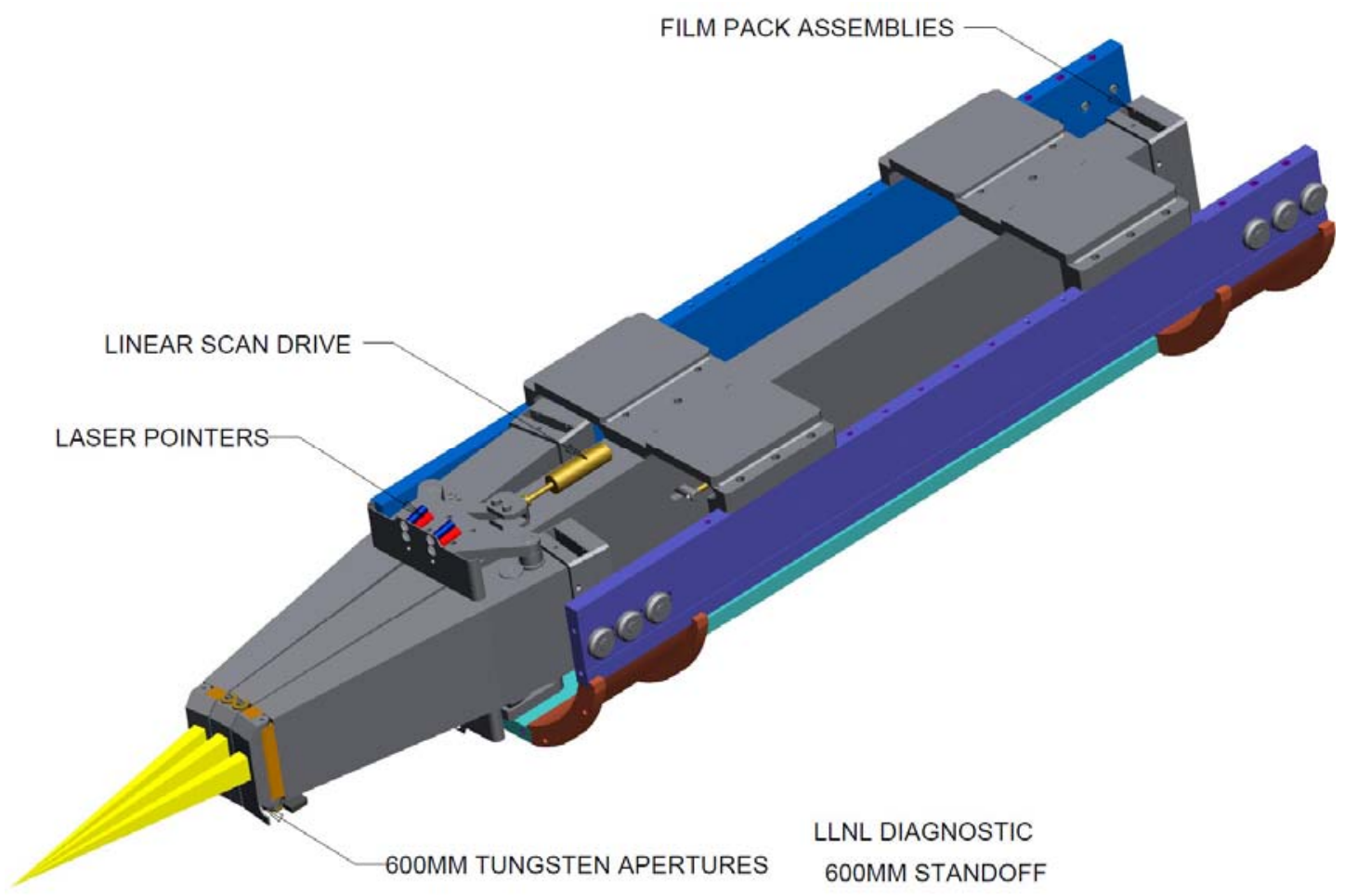

Fig. 1. Solid model illustration of the instrument deployed in a DIM and consisting of three spectrometer channels.

In order to reduce costs and to promote simplicity of construction, similar geometry and mechanical parts were used for each spectrometer channel where possible. All three spectrometer channels utilize the same size aluminum extrusion for their bodies, the same nosecone geometry, and the same image plate cassette size. 


\section{b) Spectrometer Parameters and Resolving Powers at $600 \mathrm{~mm}$ and $1000 \mathrm{~mm}$ Standoff}

The spectrometer design parameters are constrained by the dimensions of the DIM and are listed in Table 1:

\begin{tabular}{|l|c|c|c|}
\hline Channel & $\# 1$ & $\# 2$ & $\# 3$ \\
\hline Crystal & $(10-10)$ & $(10-11)$ & $(10-11)$ \\
\hline 2d $(\mathrm{nm})$ & 0.8512 & 0.6684 & 0.6684 \\
\hline Thickness $(\mathrm{mm})$ & 0.1 & 0.25 & 1 \\
\hline ROC $(\mathrm{mm})$ & 119 & 254 & 965 \\
\hline Energy Coverage (keV) & $6-20$ & $18-120$ & $50-300$ \\
\hline Detector limited RP & $240-70$ & $220-30$ & $300-30$ \\
\hline Crystal to slit distnce (mm) & & & \\
\hline \multicolumn{1}{|c|}{$600 \mathrm{~mm}$ standoff } & 54.1 & 104.8 & 267.4 \\
\hline $1000 \mathrm{~mm}$ Standoff & 56.2 & 112.7 & 325.5 \\
\hline
\end{tabular}

Listed in Table 2 for each channel and standoff distance are the calculated distances on the crystal and on the IP of the rays having the low energy and the high energy limits. Also listed are the demagnification values along the spectral line when a knife-edge aperture is placed across the crossover slit.

\begin{tabular}{|c|c|c|c|c|c|}
\hline $\begin{array}{c}\text { Spectrometer } \\
\text { No. }\end{array}$ & $\begin{array}{c}\text { Standoff } \\
\text { Distance }(\mathrm{mm})\end{array}$ & $\begin{array}{c}\text { Energy } \\
(\mathrm{keV})\end{array}$ & $\begin{array}{c}\text { Distance on } \\
\text { Crystal }(\mathrm{mm})\end{array}$ & $\begin{array}{c}\text { Distance on } \\
\text { IP }(\mathrm{mm})\end{array}$ & $\begin{array}{c}\text { Demagnification } \\
\text { using a Pinhole } \\
\text { Type Slit }\end{array}$ \\
\hline 1 & 600 & 6 & 24.1 & 28.9 & $1 / 10$ \\
\hline 1 & 600 & 20 & 7.2 & 8.7 & $1 / 10$ \\
\hline 1 & 1000 & 6 & 25.8 & 28.9 & $1 / 16$ \\
\hline 1 & 1000 & 20 & 7.7 & 8.7 & $1 / 16$ \\
\hline & 600 & 18 & 18.4 & 26.2 & $1 / 4.7$ \\
\hline 2 & 600 & 120 & 2.8 & 4.0 & $1 / 4.7$ \\
\hline 2 & 1000 & 18 & 20.9 & 26.2 & $1 / 7.9$ \\
\hline 2 & 1000 & 120 & 3.1 & 4.0 & $1 / 7.9$ \\
\hline 2 & & & & & $1 / 1.8$ \\
\hline & 600 & 50 & 13.7 & 35.8 & $1 / 1.8$ \\
\hline 3 & 600 & 300 & 2.3 & 6.0 & $1 / 2.0$ \\
\hline 3 & 1000 & 50 & 18.2 & 35.8 & $1 / 2.0$ \\
\hline 3 & 1000 & 300 & 3.0 & 6.0 & \\
\hline
\end{tabular}




\section{c) Mechanisms for Changing from $600 \mathrm{~mm}$ to $1000 \mathrm{~mm}$ Standoff}

The longitudinal axis of the highest-energy central spectrometer channel \#3 is aligned to the target by manipulating the DIM, and the axes of the two lower-energy outboard spectrometers \#1 and \#2 must be re-directed to the target when changing the standoff distance. This is accomplished by tilting (in the horizontal plane) the two outboard channels with respect to the central channel using a drive mechanism. In addition, when changing from one standoff distance to another, as indicated in the above Table 1 it is necessary to move inside each of the three spectrometers the crossover slits which are located behind the crystals. The movements of the crossover slits in the two low-energy channels \#1 and \#2 are over the small distance $2.1 \mathrm{~mm}$ and $7.9 \mathrm{~mm}$, respectively, while the distance for the high-energy channel \#3 is much larger, $58.1 \mathrm{~mm}$. Finally, it is also necessary to change the widths of the three entrance apertures at the front of the nosecones when changing the standoff distance.

In order to reduce cost, it is desirable to minimize the number of in-vacuum motors. Novel mechanisms were designed that accomplish the complex and precise motions using three motors, the first motor to tilt the two outboard channels and to move their two slits over small distances $(2.1 \mathrm{~mm}$ and $7.9 \mathrm{~mm})$, the second motor to alter the slit distance of the high-energy central spectrometer over a larger range $(58.1 \mathrm{~mm})$, and the third motor to move the three entrance apertures. Rather than physically moving the massive slit assembly of the high-energy channel over such a large distance, which would significantly alter the center of mass of the instrument, two slit assemblies are provided (one for $600 \mathrm{~mm}$ standoff and one for $1000 \mathrm{~mm}$ standoff) and are adjusted by a novel mechanism.

As shown in Fig. 1, a linear drive motor is hard-mounted to the top of the central spectrometer body, and this motor tilts the two outer spectrometer channels with respect to the central channel. The motor drives a yolk that spreads the back ends of the two outboard spectrometers as they pivot against hinges at the front ends. The outboard spectrometers are driven against a hard stop for both positions as illustrated in Fig. 2, and a limit switch signals the scan drive motor when the desired angle has been reached.

While this motion is taking place, the slits and aperture doors in the two outboard spectrometers are being positioned as well. As illustrated by the mechanism in Fig. 3, the slits are driven with a rack and spur gear assembly using the gear ratios to accurately position the two slits. Rather than using ball bearings which raises a host of lubrication and out-gassing issues in vacuum, sleeve bearings are implemented, thereby utilizing approved materials. The outer $600 \mathrm{~mm}$ aperture doors are also opened using a spur gear and rack assembly hard mounted to the center spectrometer from underneath. 


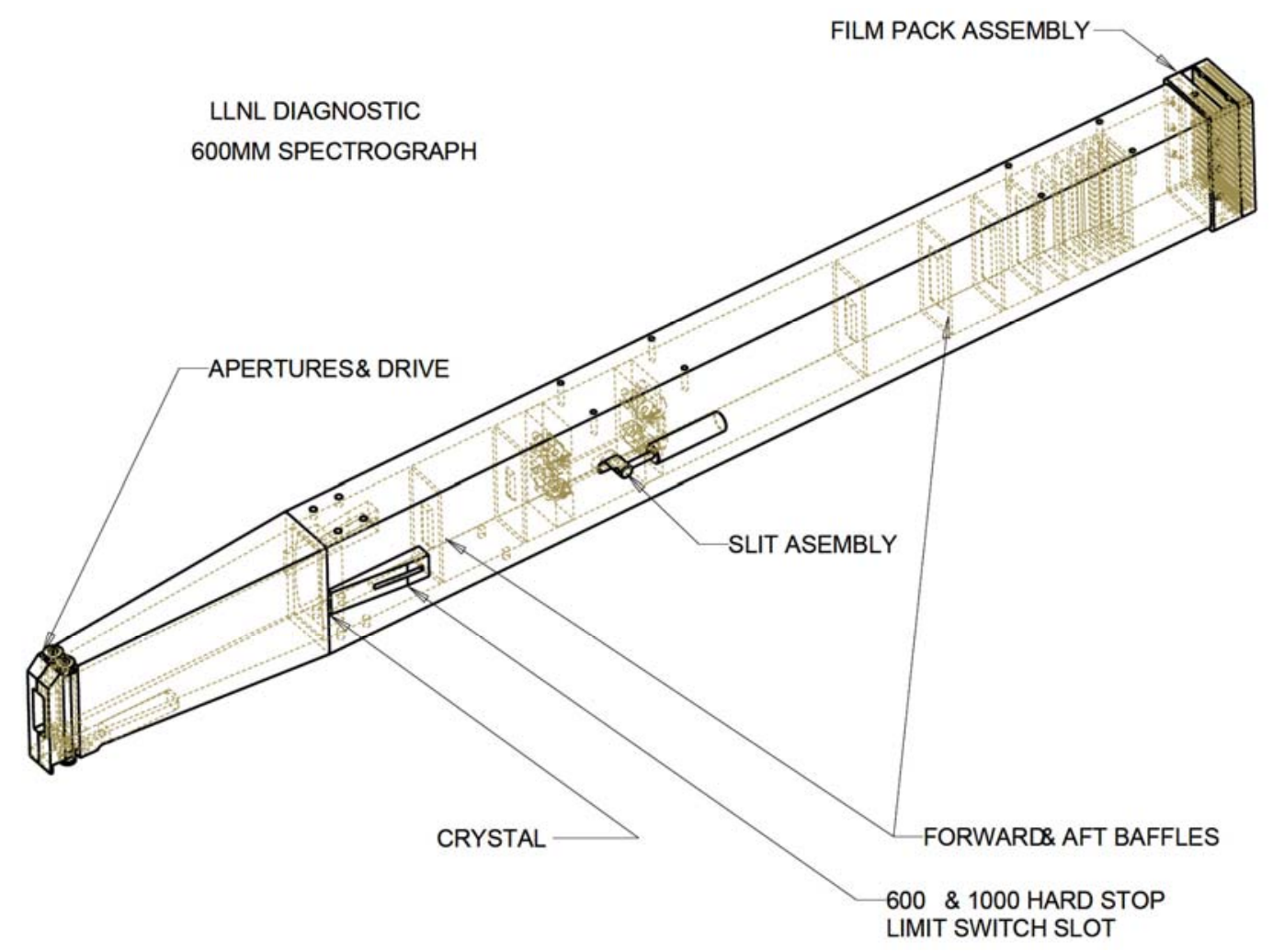

Fig. 2. View of the high-energy central spectrometer illustrating the drive motors and the forward and aft baffles.

Fig. 3. Mechanism for tilting the two outboard spectrometer channels and for moving their two slits.

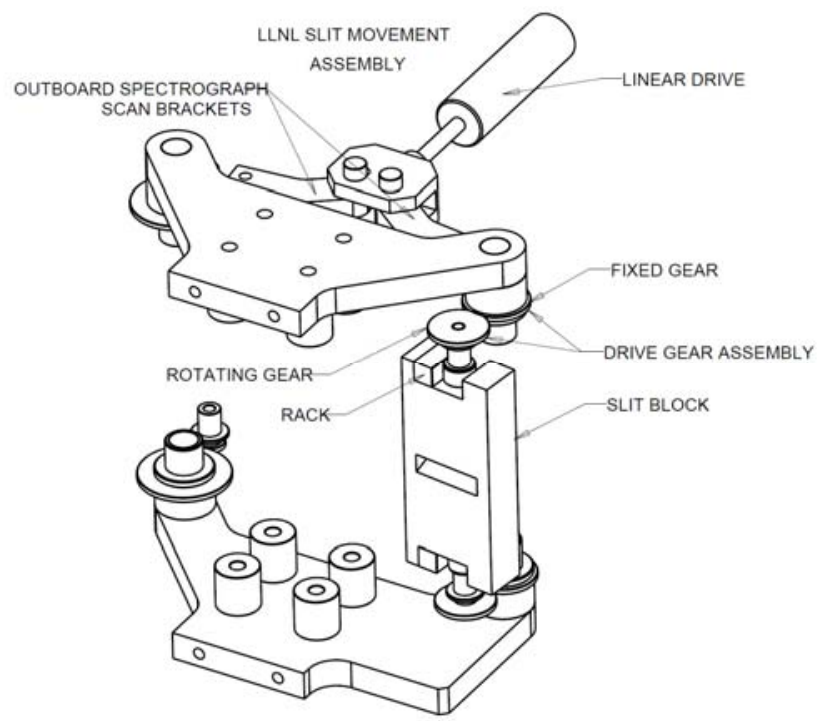


The second linear scan drive motor is used to change the slit position in the center or fixed spectrometer \#3. As illustrated in Fig. 4, this mechanism uses a linear rack to drive four gear segments attached to the four slit halves, and this arrangement also acts to "lock" the slits open or closed regardless of the instruments orientation. The concept of opening and closing two massive slits without translating them relative to the channel \#3 crystal is made necessary because of the large change in slit distance $(58.1 \mathrm{~mm})$ which if translated would significantly affect the instrument center of gravity and moment.

Fig. 4. Mechanism for altering the position of the high-energy channel slit from the front position to the back position.

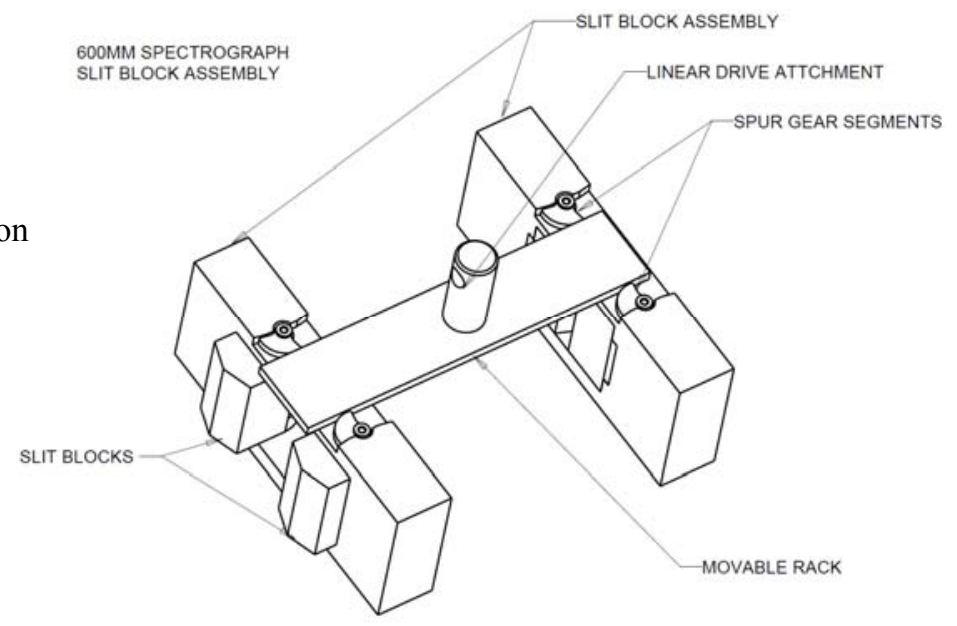

A similar concept is used to open and close the entrance apertures on the end of the nosecones. For example, the center spectrometer has a split $600 \mathrm{~mm}$ aperture door that is opened by a third linear drive motor, illustrated in Fig. 5, thereby exposing the $1000 \mathrm{~mm}$ entrance aperture.

Fig. 5. Cut-away view showing the slit drive and the aperture drive motors.

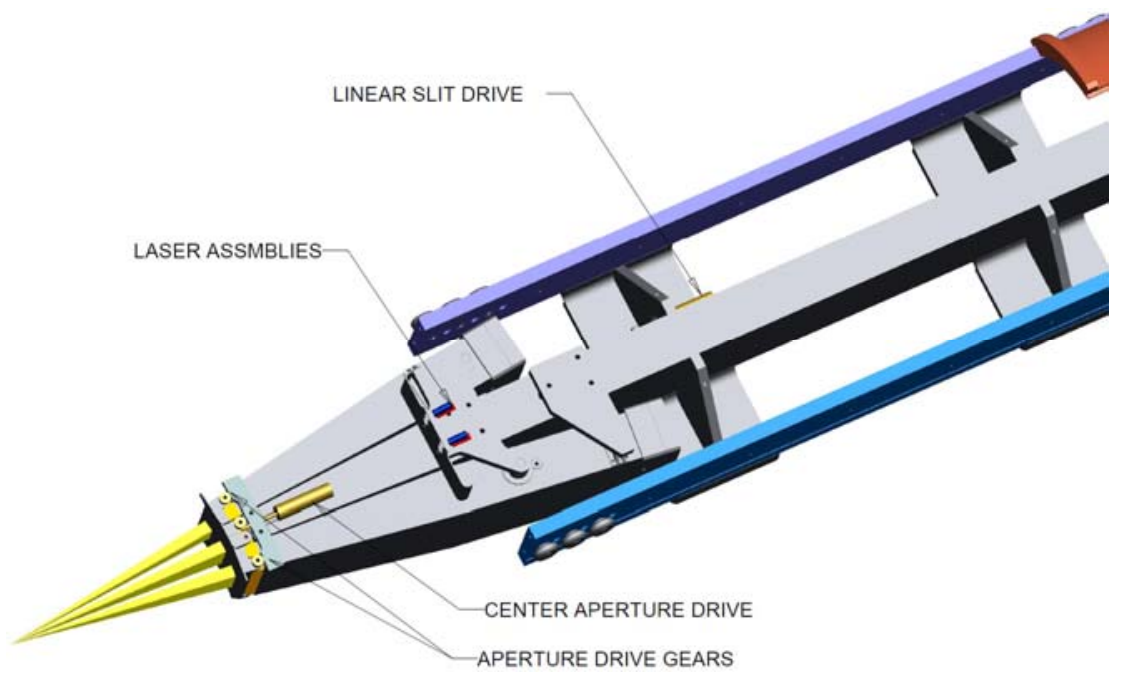




\section{d) Spectrometer Orientation, Alignment, and Pointing}

The TRI-SPEC instrument is composed of three independent spectrometer units each with its own laser alignment system. The optical alignment of the components of each spectrometer is accomplished using a laboratory $\mathrm{x}$-ray source. This includes the alignment of the front aperture, crystal, crossover slit, and detector to the axis of the spectrometer. Once a spectrometer unit is aligned to the source, the spectrometer's laser pointing system (or other pointing system specified by LLNL and TBD) is aligned to the source for each standoff distance. Then when moving from one standoff distance to another using the mechanisms described above, the alignment and pointing are checked by recording $\mathrm{x}$-ray spectra from the $\mathrm{W}$ laboratory source $(\mathrm{K}$ or L lines appear in each of the three spectrometer channels). When deployed in the DIM, the entire TRI-SPEC instrument, consisting of the three pre-aligned spectrometer units, is pointed to the target using the DIM x-y motions and the alignment/pointing lasers.

Owing to the widths of the spectra on the two sides of the spectrometer axes, the overall widths of the spectrometers (when including an additional 1" of shielding on each side) would exceed the allowed width of an instrument deployed in the DIM boat. Thus as shown in Fig. 1, the spectrometers are mounted side by side with the spectral dispersion in the vertical direction where the allowed distance within the DIM is larger than in the horizontal direction. In this orientation, the crossover slit openings in the three spectrometers are in the same horizontal direction. When aligning the instrument to the target, the most stringent alignment requirement is in the vertical (dispersion) direction, and this alignment is accomplished by moving the DIM in the vertical direction. Since the crossover slits are long in the horizontal direction, the DIM pointing in the horizontal direction is much less sensitive.

One can envision a need for each spectrometer to observe a particular part of the source, such as a backlighter target, along the horizontal direction. This could be accomplished by placing a knife edge in front the crossover slit, thereby providing moderate spatial resolution along the horizontal direction. The de-magnification in such an arrangement will vary between 1/2 to 1/16 depending on the spectrometer channel and the standoff distance (see Table 2).

\section{e) Detector Background Reduction}

Owing to the apertures at the front of the nosecone, the septum and apertures in front of the crystal, and the crossover slit, there is no direct line of sight from the source to the detector. Thus the desired rays that are diffracted by the crystal can pass through the slit and reach the detector, while undiffracted rays that might cause detector background do not pass directly through the slit. However, energetic x-rays and gamma-rays, as well as energetic electrons, can scatter from surfaces inside the target chamber, DIM, and spectrometer and can reach the detector. Finally, 
energetic neutrons can pass directly through the radiation shielding to the detector, but we have been told the TRI-SPEC instrument would not be deployed on neutron producing shots and we therefore do not consider neutron shielding. Thus we consider only shielding against energetic radiation and electrons.

The shielding is initially designed by assuming that the most intense radiation and electron fluxes are coming from the general direction of TCC. These fluxes can be divided into a component diverging in the vertical plane (perpendicular to the crossover slit and in the plane of the spectrometer dispersion) and a component diverging in the horizontal plane. The spectrometer apertures, septum, and narrow crossover slit (which are manufactured from thick tungsten) will efficiently block off-axis stray radiation in the vertical direction.

When considering the radiation coming from the front of the spectrometer and diverging in the horizontal direction, parallel to the spectrometer crossover slit, the situation is different. Owing to the length of the crossover slit, radiation coming in a narrow cone from the general direction of TCC is able to pass through the crossover slit and strike the sides of the spectrometer's back section (the section that is between the crossover slit and detector) and from there to scatter onto the detector. The geometry of the baffles that were designed for another spectrometer, the Gamma Crystal Spectrometer (GCS) fielded at EP, is illustrated in Fig. 6. Similar baffles were designed for TRI-SPEC and are illustrated in Fig. 2. The baffling systems consists of baffles forward of the crossover slit (between the crystal and slit) and aft of the slit (between the slit and the detector).

Radiation coming from the side of the spectrometer and into the back section, without scattering through the front crystal and slit assemblies, can also be divided into two components, a component that strikes the interior right and left walls and a component that strikes the interior top and bottom walls. When reaching the detector, these side rays typically have larger scattering angles from the walls compared to the rays coming from the front of the spectrometer and scattering through the crystal and slit assemblies, and the side rays are efficiently blocked by the baffling system designed for the front rays.

Since the photon energy decreases with each scatter event, the energy distribution of the scattered radiation reaching the detector is lower than the original radiation from the target. Thus the multiple baffle plates are each manufactured from relatively thin material, $2 \mathrm{~mm}$ thick phosphor bronze, and each baffle plate effectively attenuates $\mathrm{E}<70 \mathrm{keV}$ radiation.

The scattered radiation reaching the detector from the direction behind the detector can be radiation scattered from the back wall of the spectrometer or target chamber or can be radiation that passed through the detector and back-scattered from the end of the spectrometer. This radiation is eliminated by a Soller slit assembly illustrated in Fig. 7 that effectively attenuates the radiation coming from behind the detector. 

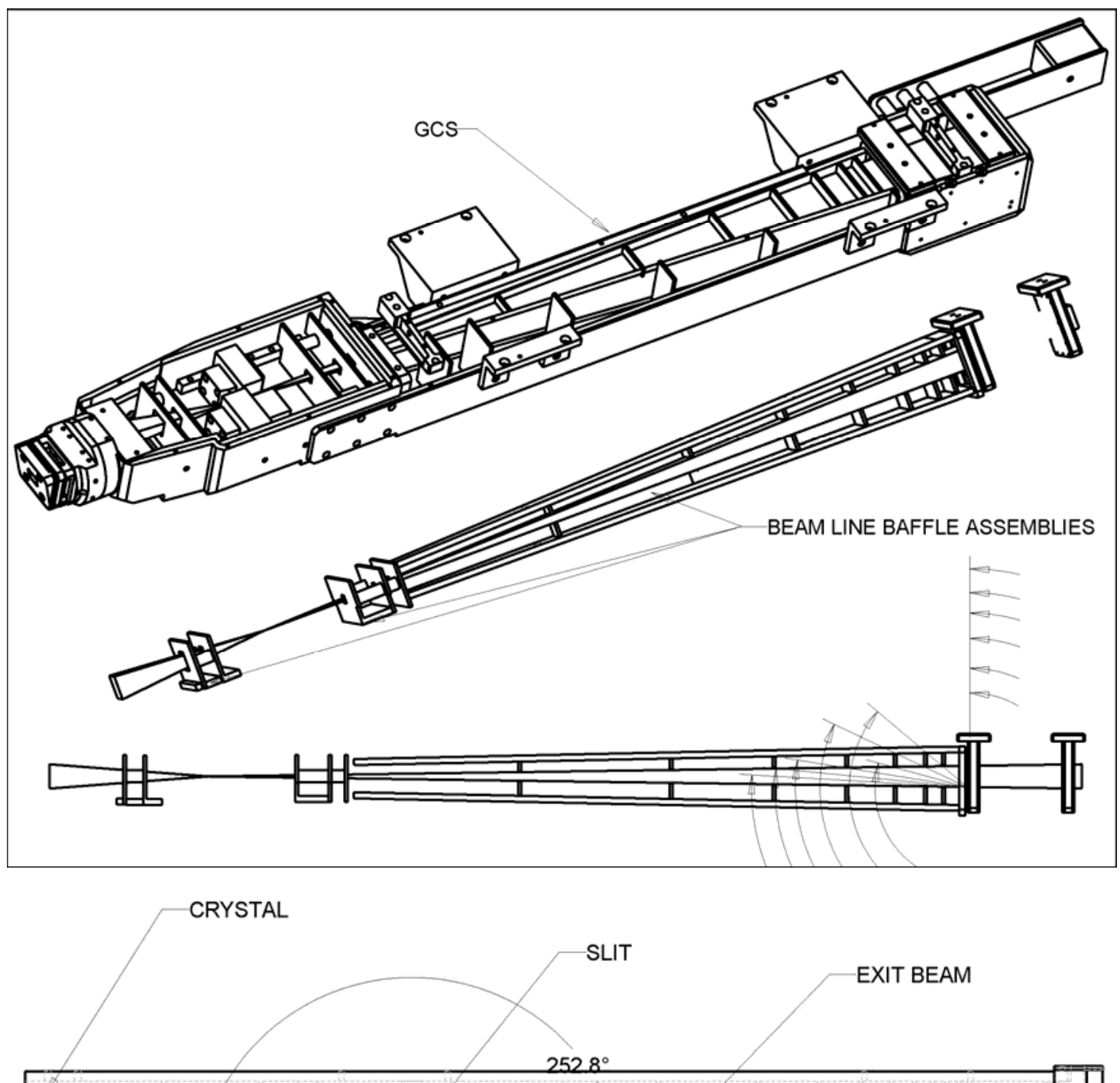

EXIT BEAM

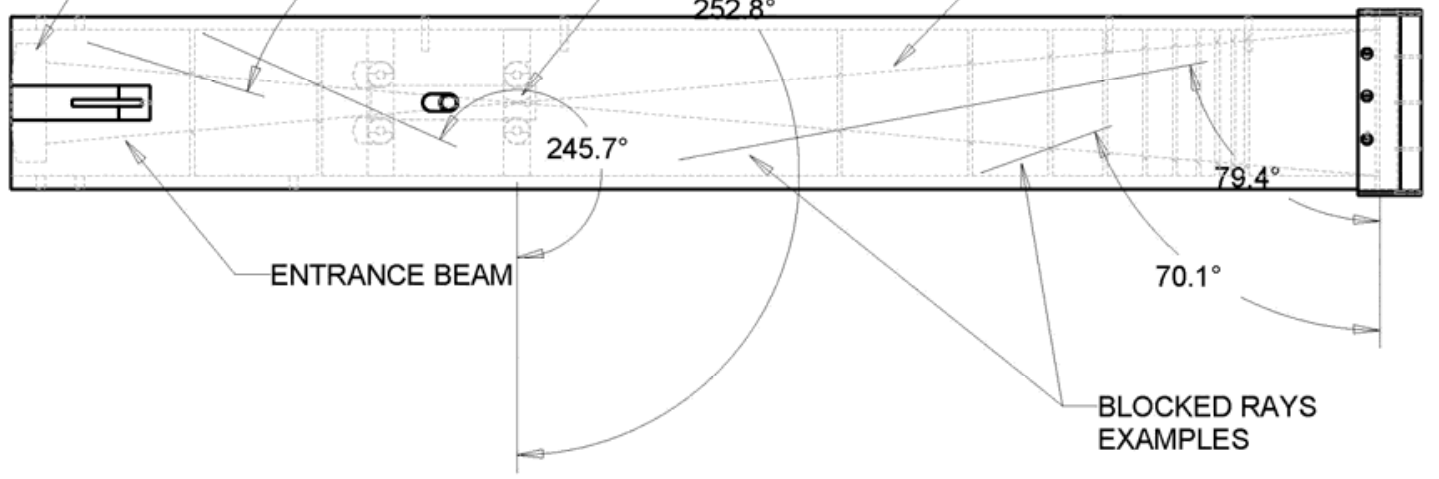

$\pi$

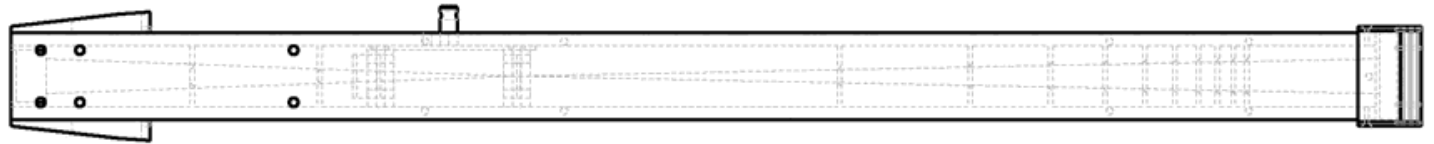

Fig. 6. The baffling system designed for the GCS spectrometer fielded at EP. 


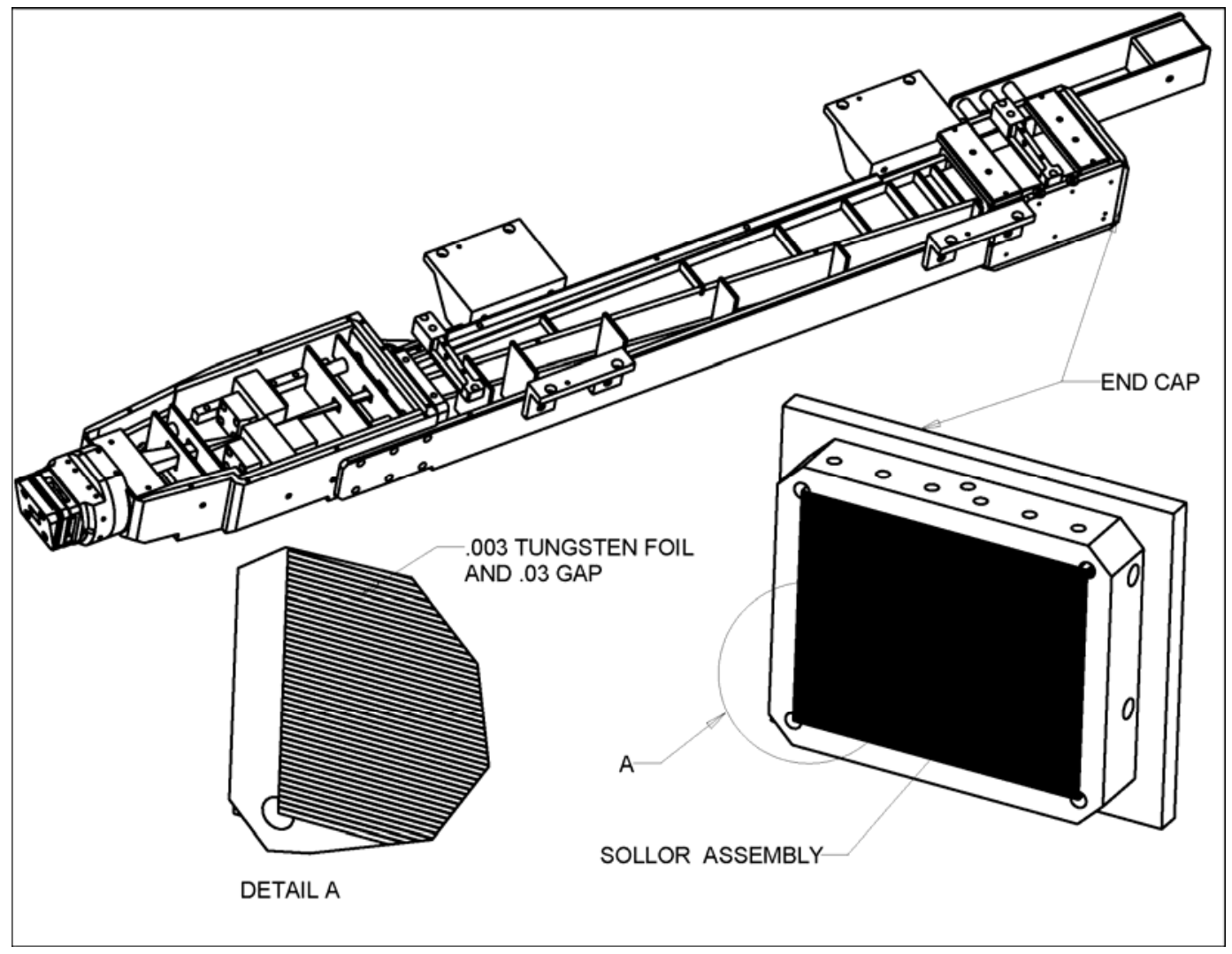

Fig. 7. The Soller slit assembly for mitigating back-scattered rays from reaching the detector.

\section{f) Absolute Calibration of the Spectrometer Sensitivity}

The absolute sensitivity of the TRI-SPEC instrument can be calibrated using a NIST calibrated source. The photon fluences are shown in Fig. 8 in the range $20 \mathrm{keV}$ to $300 \mathrm{keV}$. The bandpass of each fluence is established by attenuation filters on the low energy end and by the peak kilovoltage on the high energy end. The fluence values are accurately measured by proportional counters and by accounting for scattering in air path.

The individual spectrometer components can be calibrated such as the crystal diffraction efficiency and the detector sensitivity. The end to end calibration relates the detector signal to the absolutely calibrated fluence from the source. This enables the absolute measurement of the spectral emission from NIF targets in the $20 \mathrm{keV}$ to $300 \mathrm{keV}$ range. The absolute calibration can be extended to lower energies by the calculation of the crystal efficiency and detector sensitivity that are normalized by the $20-300 \mathrm{keV}$ measurements. 


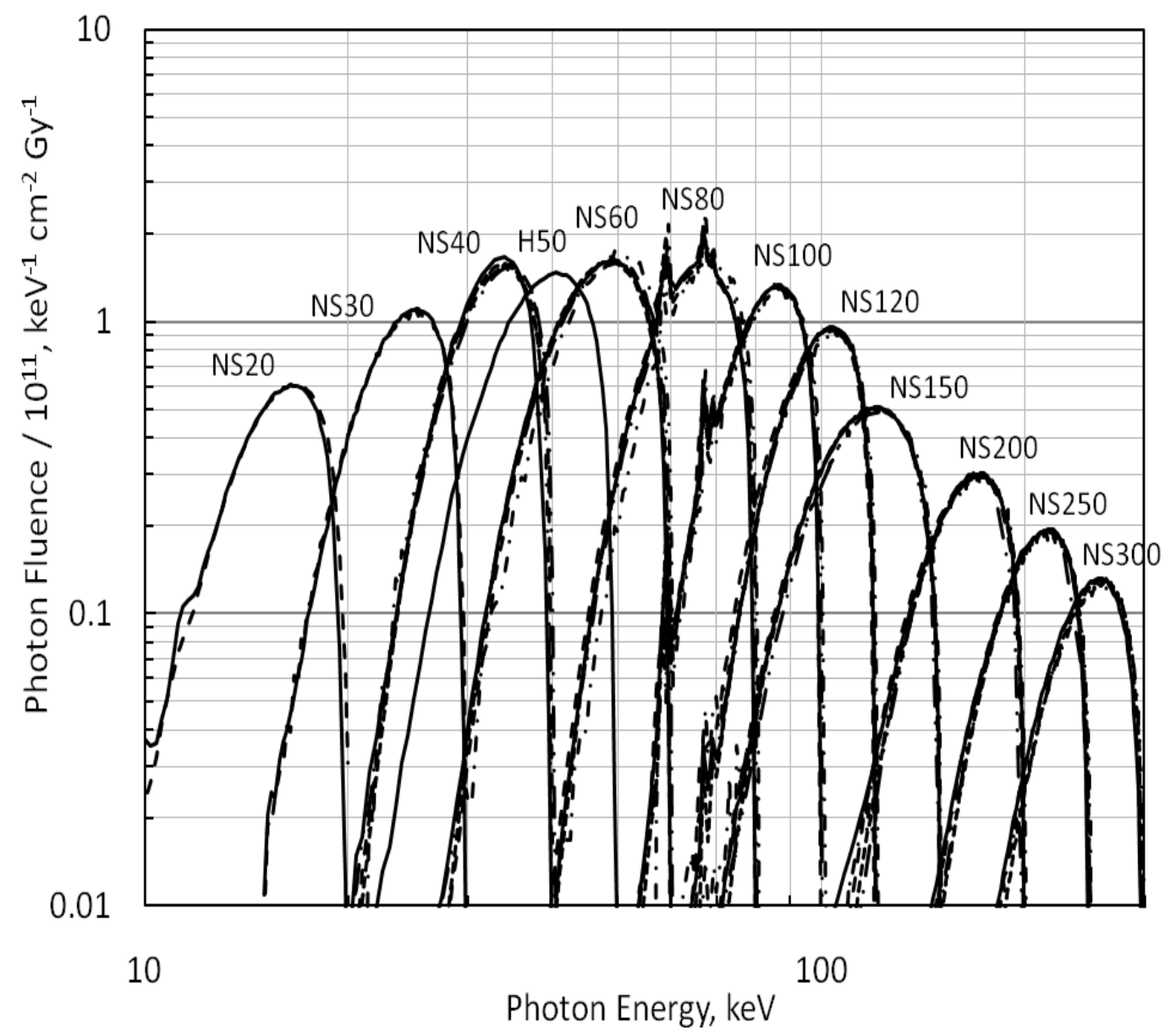

Fig. 8. The absolutely calibrated x-ray fluences from the NIST calibration source.

The separate calibration of the spectrometer components and the end to end calibration provide redundancies in the calibration procedure and therefor reliable and accurate final results. Such sophisticated and accurate calibrations are made possible by the NIST absolutely calibrated source and are not possible with detector-based calibrations that are carried out using only a calibrated detector and an un-calibrated source.

The measurements of the diffraction efficiencies of several crystals, performed in the $20 \mathrm{keV}$ to $80 \mathrm{keV}$ range at the NIST calibration facility and led by the Artep team, are included as an Attachment at the end of this report. The efficiencies were measured to 5\% absolute accuracy, and this illustrates the high accuracy and reliability of measurements carried out at the NIST calibration facility. 


\section{Low Energy and High Energy Crystal Development}

\section{a) Low Energy Crystal Development for Channel \#1}

In order to extend the energy coverage of channel \#1 down to $6 \mathrm{keV}$, it is necessary to thin the quartz crystal to below $100 \mu \mathrm{m}$ thickness. Regarding the higher energy channel \#3, the diffraction efficiency at higher energies is higher when thicker crystals are utilized. Shown in Fig. 9 is the transmittance of quartz of three thicknesses, $250 \mu \mathrm{m}$ as used in the standard Cauchois spectrometers developed by Artep and fielded at major laser facilities, $75 \mu \mathrm{m}$ as tested for lower energy coverage, and $1 \mathrm{~mm}$ as considered for the high energy channel. It is seen that the standard $250 \mu \mathrm{m}$ crystal has low transmittance below about $8 \mathrm{keV}$ while the $75 \mu \mathrm{m}$ crystal has about $25 \%$ transmittance at $6 \mathrm{keV}$ and $60 \%$ at $8 \mathrm{keV}$. Crystals with less than $75 \mu \mathrm{m}$ thickness would be fragile and difficult to bend, and they were not studied for this project.

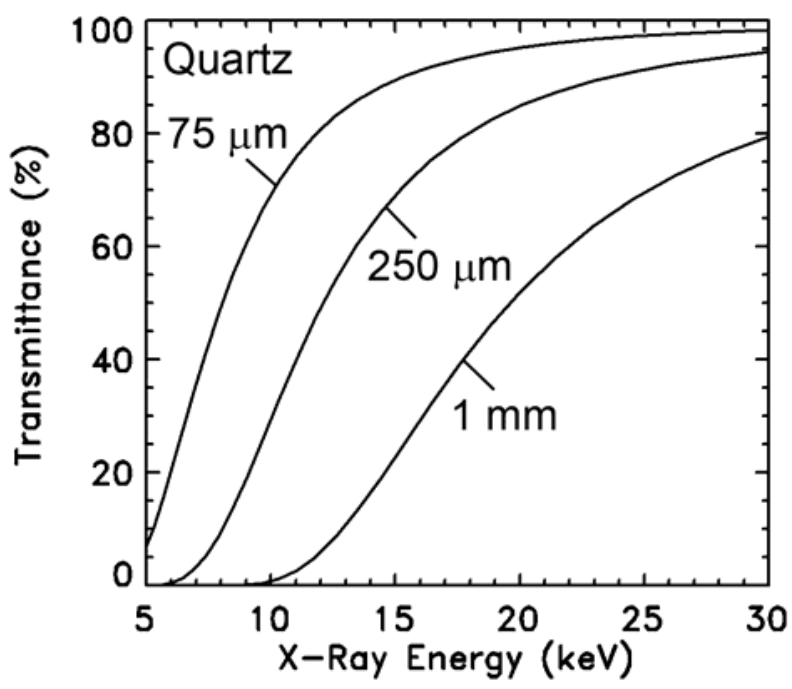

Fig. 9. Transmittance of quartz 75, 250, and $1000 \mu \mathrm{m}$ thick.

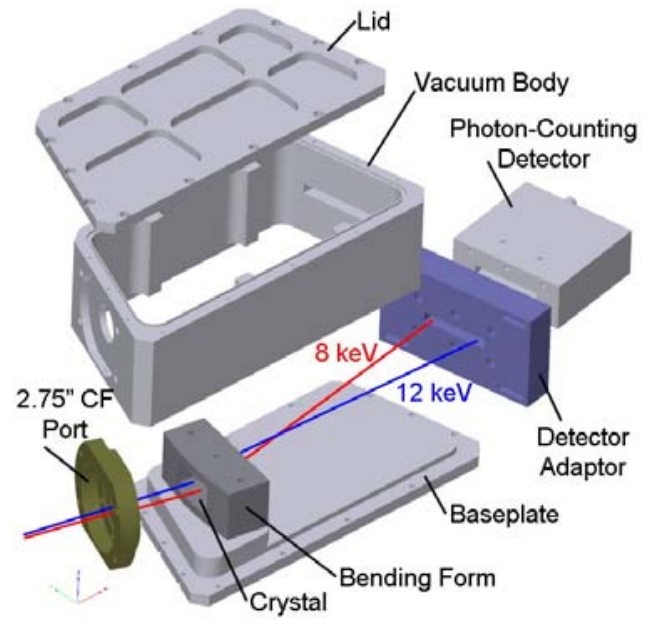

Fig. 10. Spectrometer used to test the thin crystals.

The thin crystal was evaluated using the Cauchois spectrometer shown in Fig. 10. The spectrometer and crystal orientation were initially set up for 8-12 keV coverage using a $250 \mu \mathrm{m}$ thick quartz (10-11) crystal, bent to $254 \mathrm{~mm}$ radius, and image plate detection rather than the photon-counting detector shown in Fig. 10. The spectrum from a W microfocus source is shown in Fig. 11. The numerous W L lines are identified and are well resolved. The lower-energy L $\alpha$ lines are weaker than the L $\beta$ lines because of absorption in the $250 \mu \mathrm{m}$ thick quartz crystal.

The spectra recorded using a $75 \mu \mathrm{m}$ thick quartz crystal are shown in Fig. 12. The top spectrum was recorded with the crystal in the $8-12 \mathrm{keV}$ orientation, and the bottom spectrum was recorded with the crystal rotated to cover down to lower energies. In both spectra the L $\alpha$ lines are as bright as the L $\beta$ lines owing to the increased transmittance of the $75 \mu \mathrm{m}$ crystal as compared to the 250 $\mu \mathrm{m}$ crystal. The feature near $5.8 \mathrm{keV}$ in the bottom spectrum in Fig. 11 is the L $\gamma_{2,3}$ feature in the second diffraction order, and this illustrates the ability to cover down to the $6 \mathrm{keV}$ energy region. 
Fig. 11. The W L shell spectrum recorded by the $250 \mu \mathrm{m}$ thick quartz crystal (the image plate is shown below).

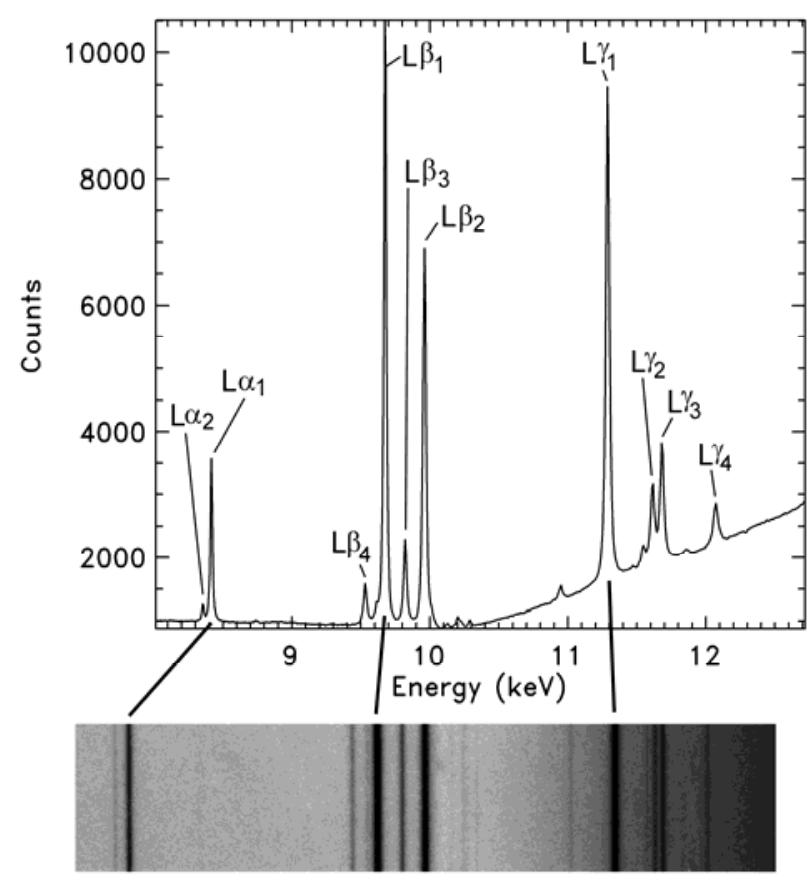

Fig. 12. The $\mathrm{W} L$ shell spectra recorded by the $75 \mu \mathrm{m}$ thick quartz crystal in the nominal $8-12 \mathrm{keV}$ orientation (top) and rotated to cover lower energies (bottom). The features appearing at $5.8 \mathrm{keV}$ in the bottom spectrum are the second order $\mathrm{L} \gamma_{2}$ and $\mathrm{L} \gamma_{3}$ lines.
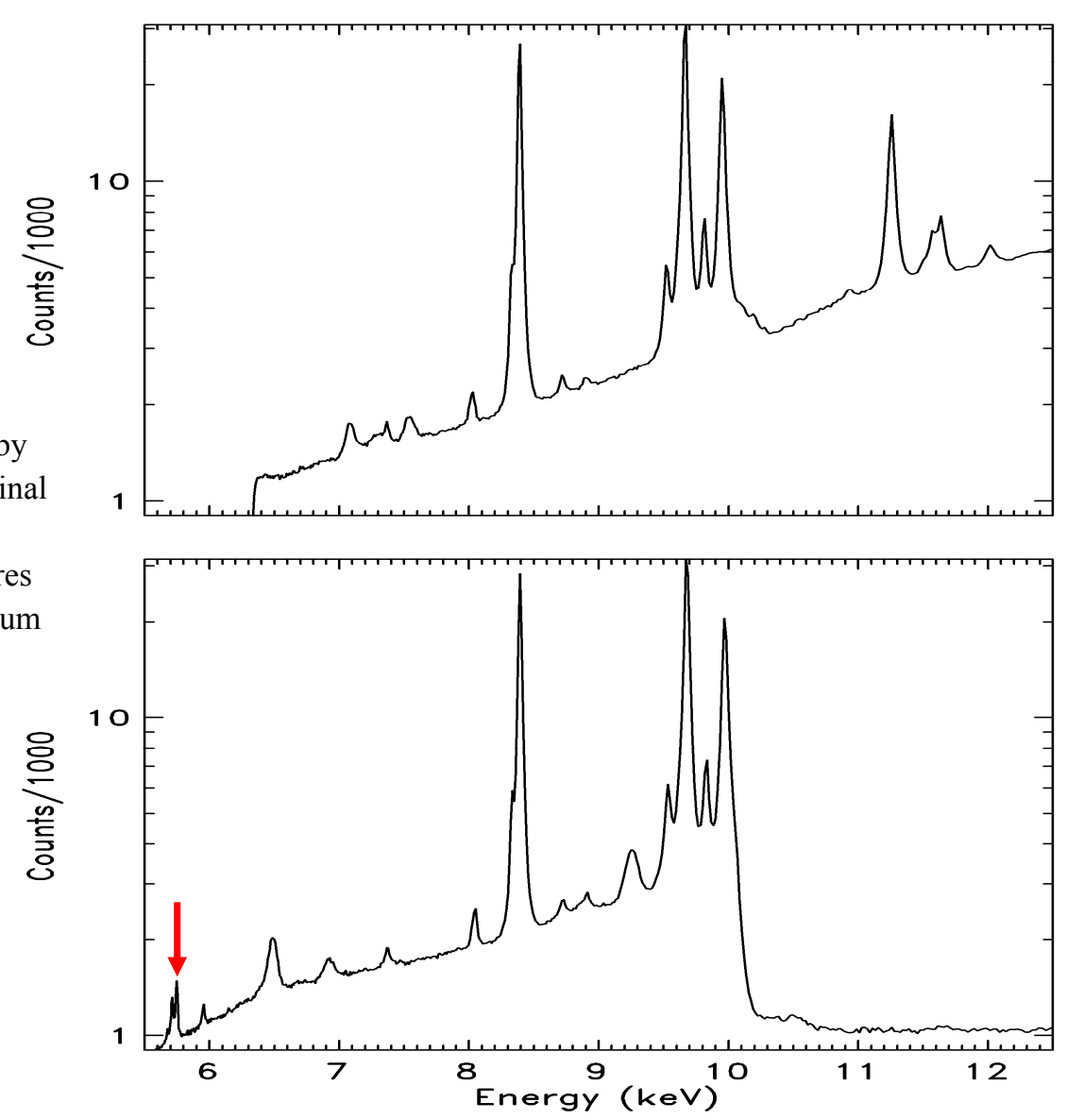


\section{b) High Energy Crystal Development for Channel \#3}

In order to record spectra up to energy $511 \mathrm{keV}$ for the study of the positron-electron annihilation radiation generated during LLNL experiments using intense picosecond laser irradiation of gold targets, it is necessary to utilize a high dispersion crystal to move the spectral features farther from the spectrometer axis where scatter radiation can obscure the spectrum. This can be accomplished by using a crystal with a small $2 \mathrm{~d}$ spacing and operating in a high diffraction order. The tradeoff is that the diffraction efficiencies in the higher orders are lower as illustrated in the Table 3 of the relative efficiencies of quartz cuts.

In addition, it is beneficial to utilize a thicker crystal which can have higher efficiency compared to a thinner crystal. However, as shown in Fig. 9, a thicker crystal cannot operate at lower energies

Table 3. Calculated relative efficiencies of quartz.

\begin{tabular}{|c|c|c|c|c|}
\hline Miller & \multicolumn{2}{|c|}{ Relative efficiency } & 2d (Ang) & \\
\hline Indices & $\mathrm{n}=1$ & $\mathrm{n}=2$ & $\mathrm{n}=1$ & $\mathrm{n}=2$ \\
\hline & & & & \\
\hline$(203)$ & 100 & 17 & 2.750 & 1.375 \\
\hline$(031)$ & 85 & 6 & 2.744 & 1.372 \\
\hline$(502)$ & 37 & 12 & 1.624 & 0.812 \\
\hline$(503)$ & 10 & 11 & 1.539 & 0.769 \\
\hline$(533)$ & 21 & 4 & 1.152 & 0.576 \\
\hline
\end{tabular}
owing to absorption.

Finally, crystals composed of high atomic number elements have higher coherent scattering cross sections (and higher diffraction efficiencies) compared to lighter elements. The tradeoff is that the incoherent (Compton) scattering is also higher and can contribute to background on the detector. In the bent-crystal Cauchois geometry, the radiation scattered from the crystal can be controlled by the narrow crossover slit which is located between the crystal and the detector, as illustrated in Fig. 2, and this is a tremendous benefit of the Cauchois spectrometer design.

These three aspects of developing crystals for use up to $511 \mathrm{keV}$ were studied: (1) High dispersion, (2) Increased crystal thickness, and (3) High atomic number.

\section{(1) $\quad$ High dispersion}

The quartz (101) cut is commonly used in our Cauchois spectrometers because it has relatively high diffraction efficiency and is readily available with large area and at a reasonable cost. The higher order quartz cuts listed in Table 3 have smaller $2 \mathrm{~d}$ spacings and smaller relative efficiencies, but they do have the benefit of higher dispersion. The quartz (203), (031), and (502) crystals were studied.

Shown in Fig. 13 is a comparison of W spectra recorded with same source conditions and with the quartz (203) and (301) crystals bent to $965 \mathrm{~mm}$ radii. The spectral lines are the $\mathrm{W} \mathrm{K \alpha _{1 }}$ and $\mathrm{K} \alpha_{2}$ lines (59.318 and $\left.57.982 \mathrm{keV}\right)$ and the $\mathrm{K} \beta_{1}$ and $\mathrm{K} \beta_{2}$ lines $(67.244$ and $69.101 \mathrm{keV})$. 
Compared to the (203) spectrum, the (301) spectrum has higher dispersion (because of the smaller $2 \mathrm{~d}$ spacing) and lower relative line intensity and efficiency.

Shown in Fig. 14 is a portion of a W spectrum, covering the $\mathrm{K} \beta$ lines, which was recorded with a quartz (203) crystal. The crystal was bent to a large radius ( $965 \mathrm{~mm}$ ), and the spectrum was recorded by a Hamamatsu dental x-ray CCD having relatively small $20 \mu \mathrm{m}$ pixels, both of which promoted good spectral resolution. The experimental $\mathrm{W} \mathrm{K} \beta$ spectrum is compared to a simulated spectrum, and it is seen that numerous $\mathrm{K} \beta$ lines are resolved. This illustrates the benefits of using a crystal with small $2 \mathrm{~d}$ spacing, large bending radius, and high resolution detector.

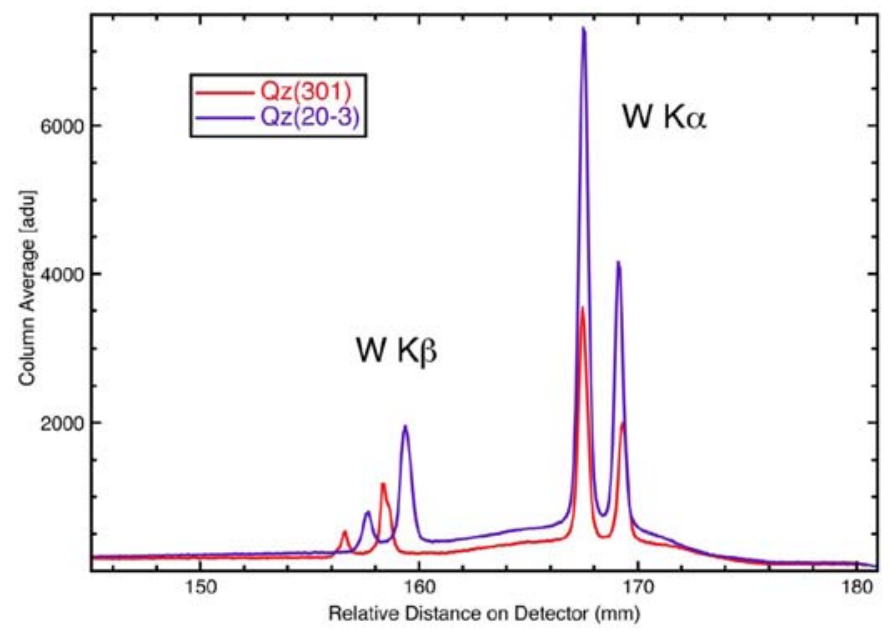

Fig. 13. Comparison of $\mathrm{W}$ spectra recorded with the same source conditions and using quartz (203) and (301) crystals.

Fig. 14. The experimental spectrum recorded using a quartz (502) crystal is shown at the right, and the simulated spectrum is shown below.

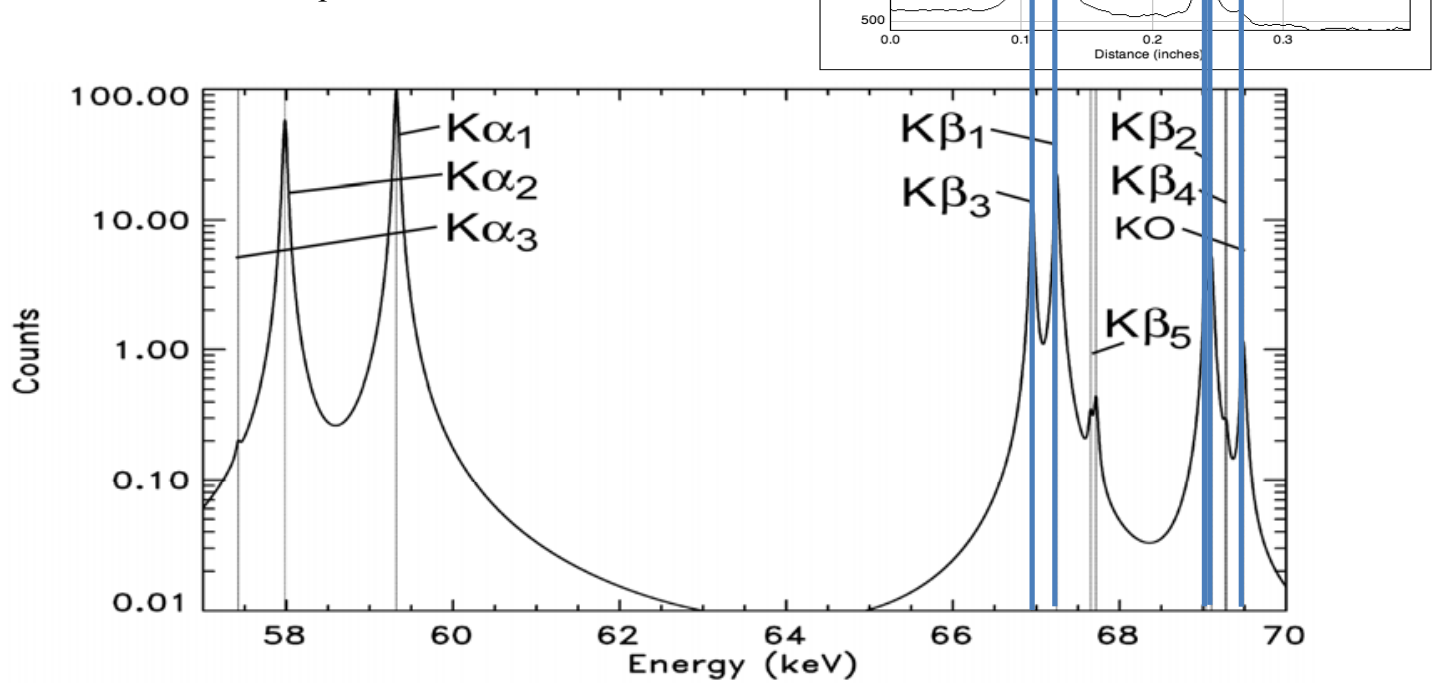




\section{(2) Increased Crystal Thickness}

The diffraction efficiency of a transmission crystal increases with the crystal thickness so long as the thickness is not sufficient to cause substantial absorption. Depending on the material properties, a thicker crystal is typically more difficult to bend than a thinner crystal. Thus there are limits on the thickness of a bent transmission crystal. The difficulty of bending a thick crystal can be mitigated if multiple thinner crystals can be layered and bent with good optical figure so that the spectral resolution is not substantially affected. This motivated the spectroscopic testing tandem bent crystals.

Shown in Fig. 15 is the W spectrum recorded by two quartz crystals that were each $250 \mu \mathrm{m}$ thick and were bent together on a form having $254 \mathrm{~mm}$ radius of curvature. For the same source conditions, the spectral lines were roughly a factor of two brighter than those from a single 250 $\mu \mathrm{m}$ thick crystal. Moreover, the spectral resolution produced by the two crystals was the same as produced by a single crystal. This demonstrates the feasibility of using multiple thin crystals, accurately bent onto a form, to provide increased efficiency equivalent to one thick crystal which may be difficult or impossible to bend onto the form.

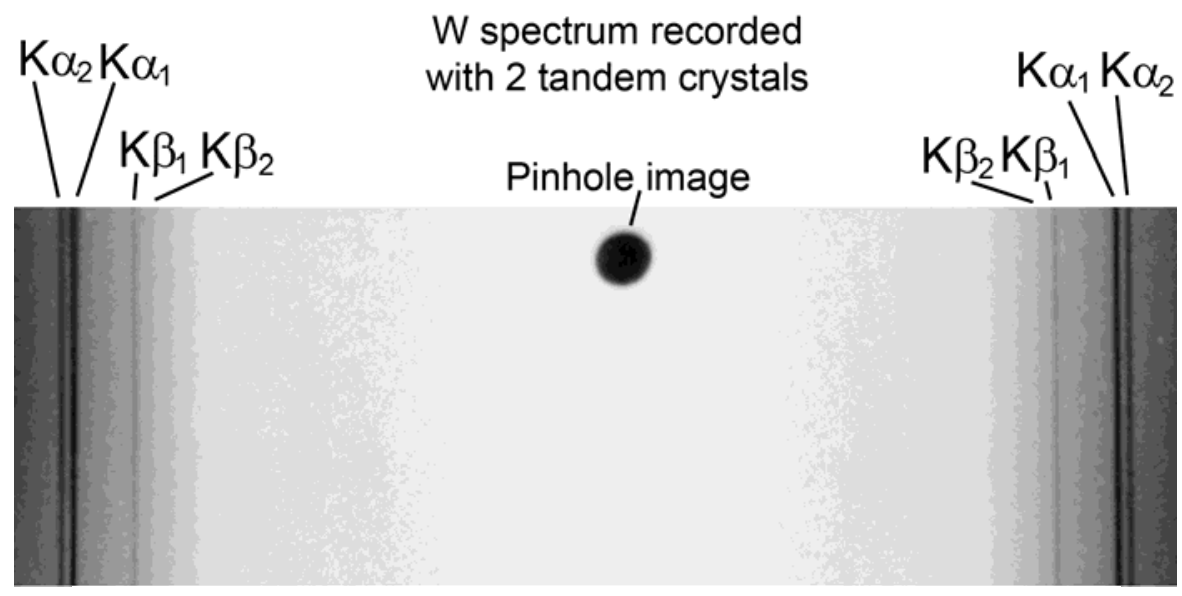

Fig. 15. W spectrum recorded by two $250 \mu \mathrm{m}$ thick quartz crystals.

\section{(2) High Atomic Number Crystals}

While custom fabricated slabs of the commonly used crystals silicon, germanium, and quartz are readily available, custom slabs of crystals composed of higher atomic number elements are not readily available. However, large commercial wafers of single-crystal (100) GaAs and InSb and with up to $1 \mathrm{~mm}$ thicknesses are readily available and were evaluated in the Laue diffraction geometry. In addition, $1 \mathrm{~mm}$ thick single-crystal (100) Au and Al crystals were procured and evaluated. 
The setup for testing the flat crystals in the Laue transmission geometry is shown in Fig. 16. The crystal and the crossover aperture were mounted on rails, and the entire assembly was mounted on an $x-y-z$ stage which was used to orient the axis of the rails to the x-ray source. The $\mathrm{W} x$-ray source is to the right in Fig. 13, and the image plate detector is to the left. The crystal mount had tip-tilt motions that were used to precisely align the crystal to be perpendicular to the axis of the rails. Then the x-rays from the source were diffracted at symmetrical location about the center of the crystal, and the inside diffracted rays of all energies converged through the crossover aperture and diverged to the detector. A septum rod was mounted at the center of the crystal and shadowed the crossover aperture, thereby blocking axial rays from reaching the detector.

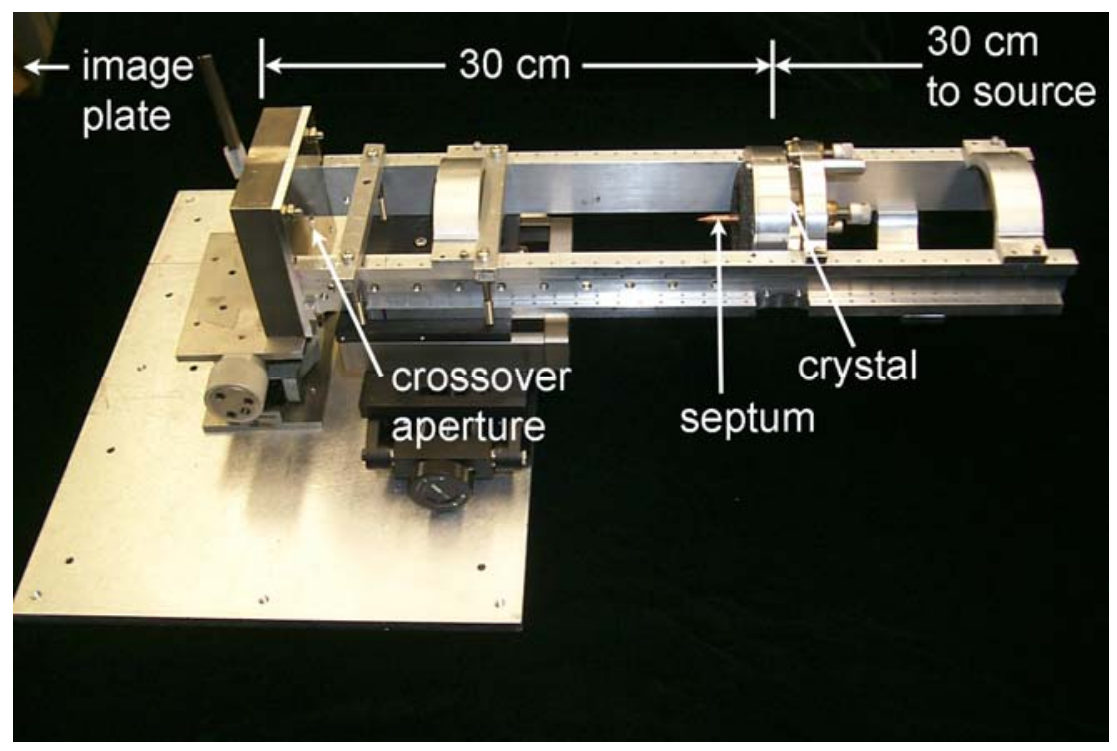

Fig. 16. Setup for testing the high atomic number crystals.

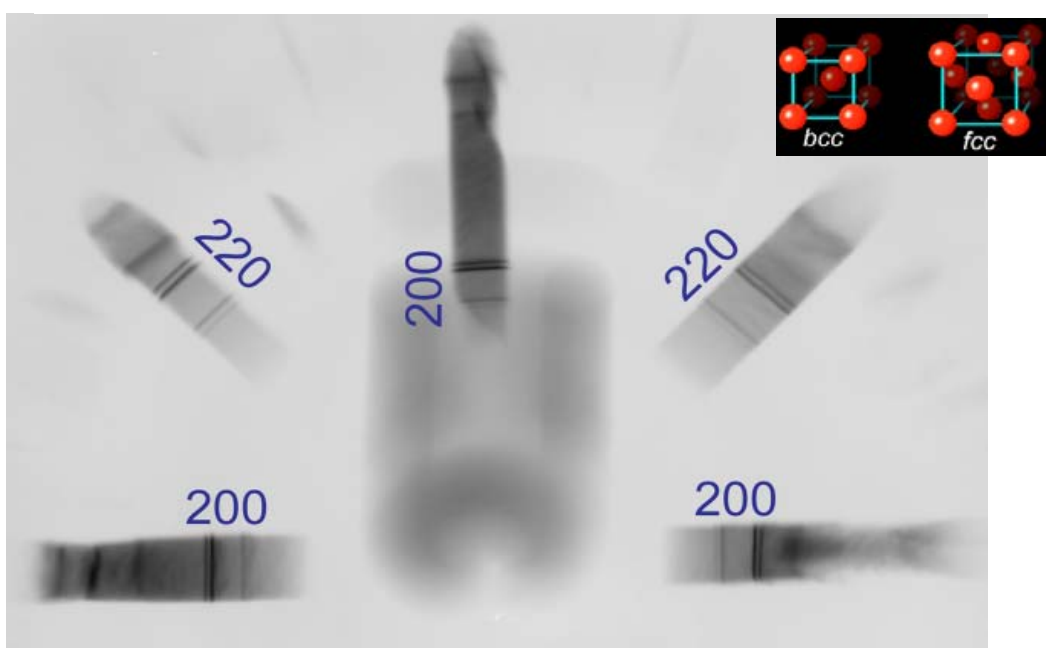

Fig. 17. Laue pattern produced by a $1 \mathrm{~mm}$ thick Al (100) crystal. 
A portion of the Laue pattern produced by a $1 \mathrm{~mm}$ thick $\mathrm{x} 20 \mathrm{~mm}$ diameter $\mathrm{Al}$ (100) crystal is shown in Fig. 17. An Au (100) crystal of the same size was also tested. The pattern is composed of radial bands of dispersed continuum radiation and superimposed $\mathrm{K} \alpha$ and $\mathrm{K} \beta$ lines, with energy increasing toward the center of the pattern. For each band, the Miller indices of the crystal planes were identified based the distance of the $\mathrm{K}$ lines from the center of the pattern, the azimuthal angular orientation of the band, and the $2 \mathrm{~d}$ spacings of the allowed diffraction by the crystal's unit cell. The Al 2d spacings are listed in the below Table 4 along with several other crystals.

\begin{tabular}{|c|c|c|c|c|c|c|}
\hline $\begin{array}{l}\text { Miller } \\
\text { Index } \\
h \ell k\end{array}$ & $\begin{array}{c}\text { Angle } \\
\text { from } 100 \\
\text { (deg) }\end{array}$ & $\begin{array}{c}\text { GaAs (z. b.) } \\
\mathrm{a}=5.653 \AA \\
2 \mathrm{~d}(\AA)\end{array}$ & $\begin{array}{c}\text { Si (cubic) } \\
a=5.431 \AA \\
2 d(\AA)\end{array}$ & $\begin{array}{c}\text { Al (fcc) } \\
\mathrm{a}=4.050 \AA \\
2 \mathrm{~d}(\AA)\end{array}$ & $\begin{array}{c}\mathrm{W}(\mathrm{bcc}) \\
\mathrm{a}=3.165 \AA \\
2 \mathrm{~d}(\AA)\end{array}$ & $\begin{array}{l}\text { Bragg Angle } \\
\text { at } 511 \mathrm{kev} \\
\text { (deg) }\end{array}$ \\
\hline 200 & 0,90 & 5.653 & forbidden & 4.050 & 3.165 & 0.439 \\
\hline 400 & 0,90 & 2.827 & 2.716 & 2.025 & 1.583 & 0.879 \\
\hline 800 & 0,90 & 1.413 & 1.358 & & & \\
\hline 220 & 45 & 3.997 & 3.840 & 2.864 & 2.238 & 0.621 \\
\hline 440 & 45 & 1.999 & 1.920 & 1.432 & 1.119 & 1.242 \\
\hline 660 & 45 & 1.332 & 1.280 & & & \\
\hline 880 & 45 & 0.999 & 0.960 & & & \\
\hline 620 & 18.4 & 1.788 & & & & \\
\hline 840 & 26.6 & 1.264 & & & & \\
\hline 1020 & 11.3 & 1.109 & & & & \\
\hline & & & & & & \\
\hline 1060 & 31.0 & 0.970 & & & & \\
\hline
\end{tabular}

The Laue pattern produced by a $1 \mathrm{~mm}$ thick GaAs (100) commercial wafer is shown in Fig. 18. The wafer is 4" in diameter, and this large area permitted the observation of larger Bragg angles and higher diffraction orders, up to (10 6 0), compared to the $20 \mathrm{~mm}$ diameter Al crystal. In addition, more orders were allowed in the bi-atomic GaAs crystal as compared to the Al crystal.

While these tests demonstrate the ability of thick $(1 \mathrm{~mm})$ commercial flat crystals to produce high-quality spectra, initial attempts to bend the thick crystals were not successful. We believe additional techniques, such as bending at elevated temperatures and using accurately machined forms that bend the crystal from both sides, rather than from one side, could be successful. In any case, the spectra that were recorded using two thinner crystals bent in tandem, as discussed in the previous Section, demonstrated the ability to record high resolution spectra without bending a 1 mm thick crystal. 


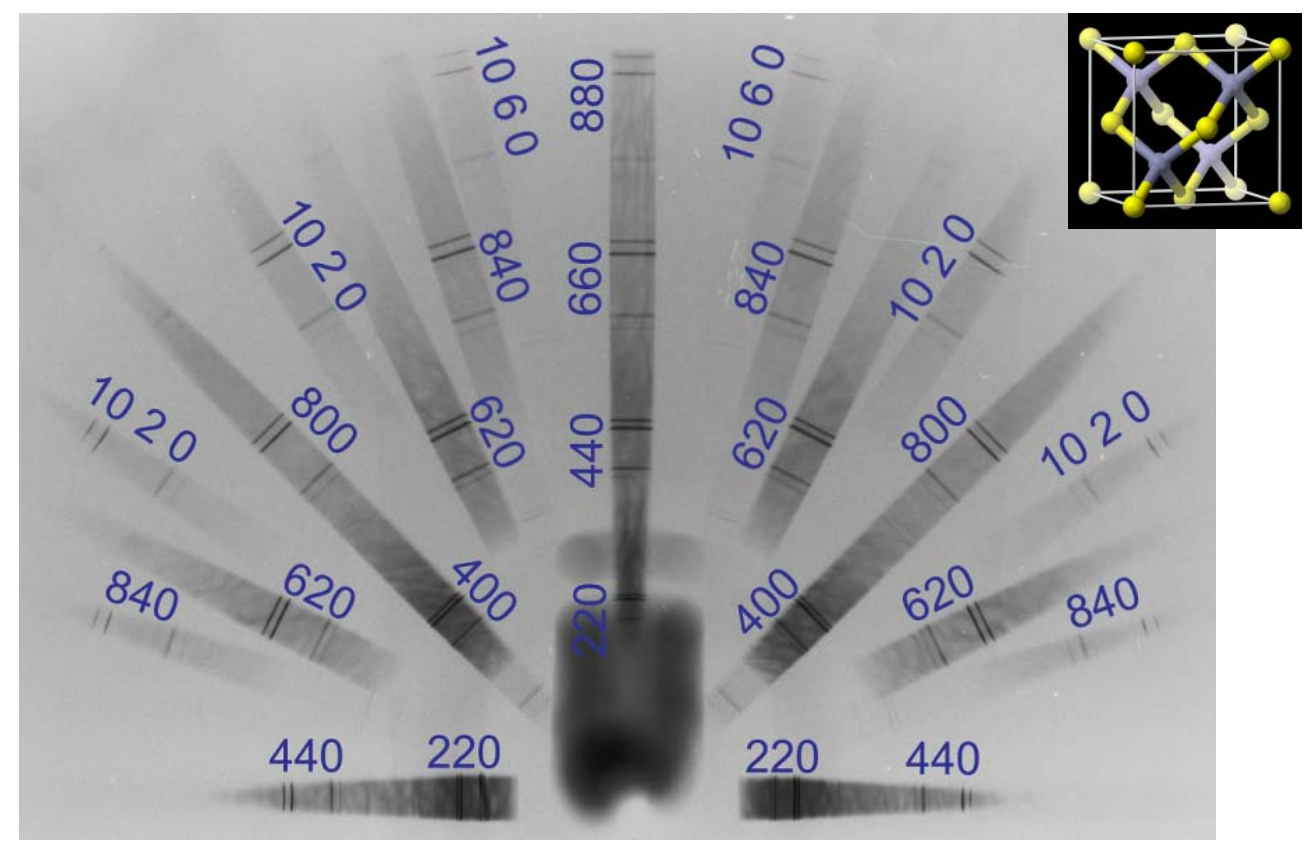

Fig. 18. The Laue pattern produced by a $1 \mathrm{~mm}$ thick GaAs (100) wafer.

\section{(c) Testing at $662 \mathrm{keV}$ using a Cs-137 Source}

The setup for testing the thick crystals at a Cs- 137 radioactive source facility, which produces mostly $662 \mathrm{keV}$ gammas, is shown in Fig. 19. The setup has the same linear geometry as the setup shown in Fig. 16 except the source to crystal and crystal to detector distances are each $1 \mathrm{~m}$ rather than $30 \mathrm{~cm}$. In the $662 \mathrm{keV}$ setup, the Cs- 137 source is inside a vertical well, and the rails holding the crystal and crossover aperture assemblies are vertical. The various apertures and shielding at the $662 \mathrm{keV}$ source were much thicker than used at the $\mathrm{W}$ source. For example, the source aperture is a hole in a 2" thick lead brick, the crossover aperture is a hole in a 1" thick tungsten block, and massive lead shielding was used around the detector.

The Cs-137 source and the image plate sensitivity were initially characterized without the Laue apparatus in place. The Cs-137 source, which had $3.25 \mathrm{Ci}$ activity $\left(1.0 \times 10^{11} 662 \mathrm{keV}\right.$ gammas/sec), was characterized using an AMPTEK X-123 photon-counting detector having a CdTe chip with $5 \mathrm{~mm} \times 5 \mathrm{~mm}$ area and $2.25 \mathrm{~mm}$ thickness. The spectra recorded with various source to detector distances are shown in Fig. 20.

Based on the source spectra recoded by the AMPTEK detector, the image plate sensitivity to 662 $\mathrm{keV}$ gammas was calibrated. Shown in Fig. 21 is a 3 minute exposure through 2" thick lead bricks and other test objects, and it was determined that the Logos image plate and scanner recorded 0.2 counts per $662 \mathrm{keV}$ incident photon. 
The Laue setup shown in Fig. 19 was installed over the Cs-137 source well, and attempts were made to record a $662 \mathrm{keV}$ spectrum on the image plate through $1 \mathrm{~mm}$ thick flat crystals. While radiation was recorded, it could not be conclusively identified as resulting from $662 \mathrm{keV}$ photons that were dispersed by the crystal. We believe it is necessary to utilize a thick bent crystal, or tandem combination of several thinner bent crystals, and to thereby focus the dispersed $662 \mathrm{keV}$ photons into the image plate detector. In addition, the shielding surrounding the image plate must be increased to prevent scattered $662 \mathrm{keV}$ photons from reaching the detector.

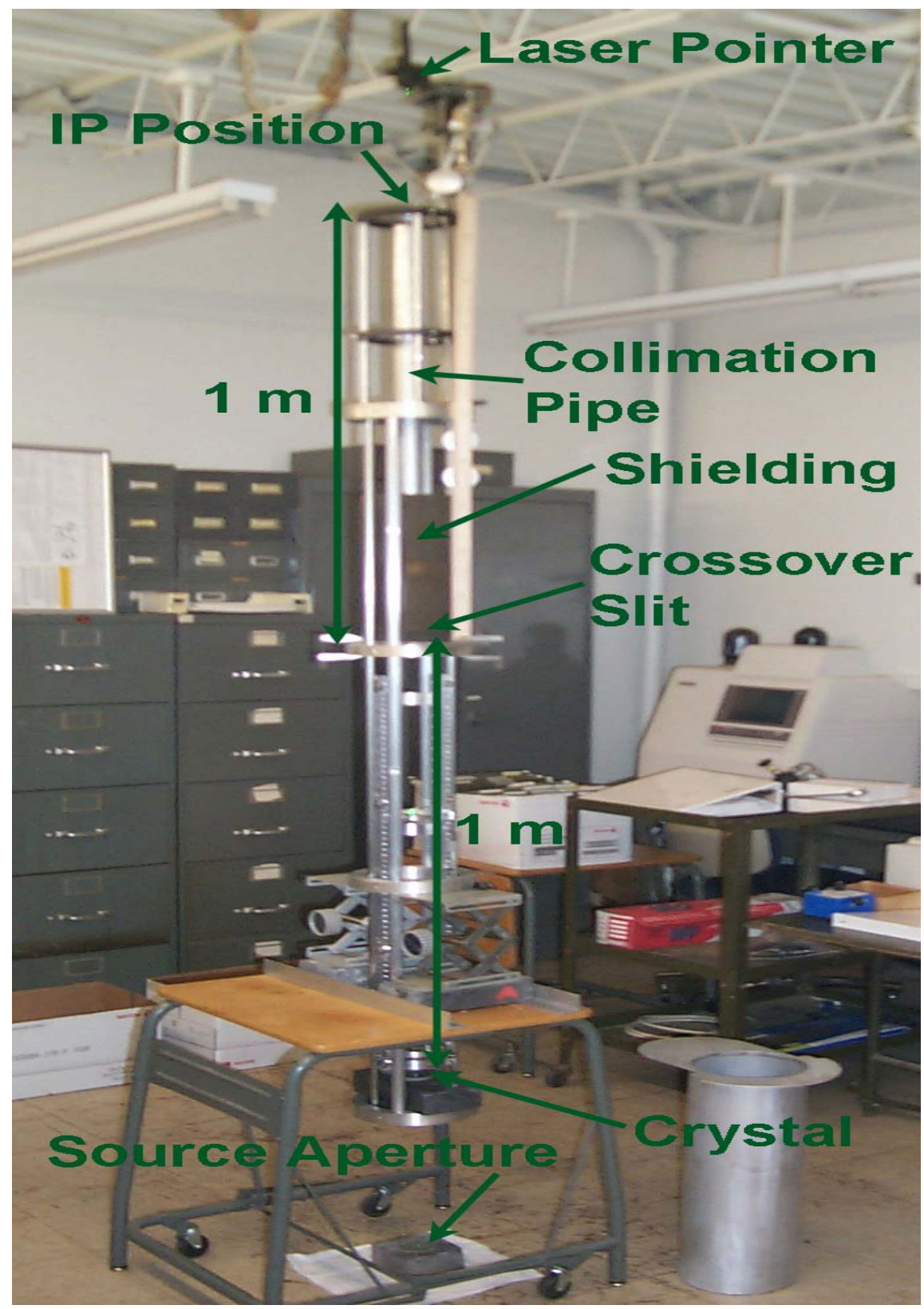

Fig. 19. Setup for testing $1 \mathrm{~mm}$ thick crystals at the $662 \mathrm{keV}$ Cs-137 source. 


\section{Photoelectric}

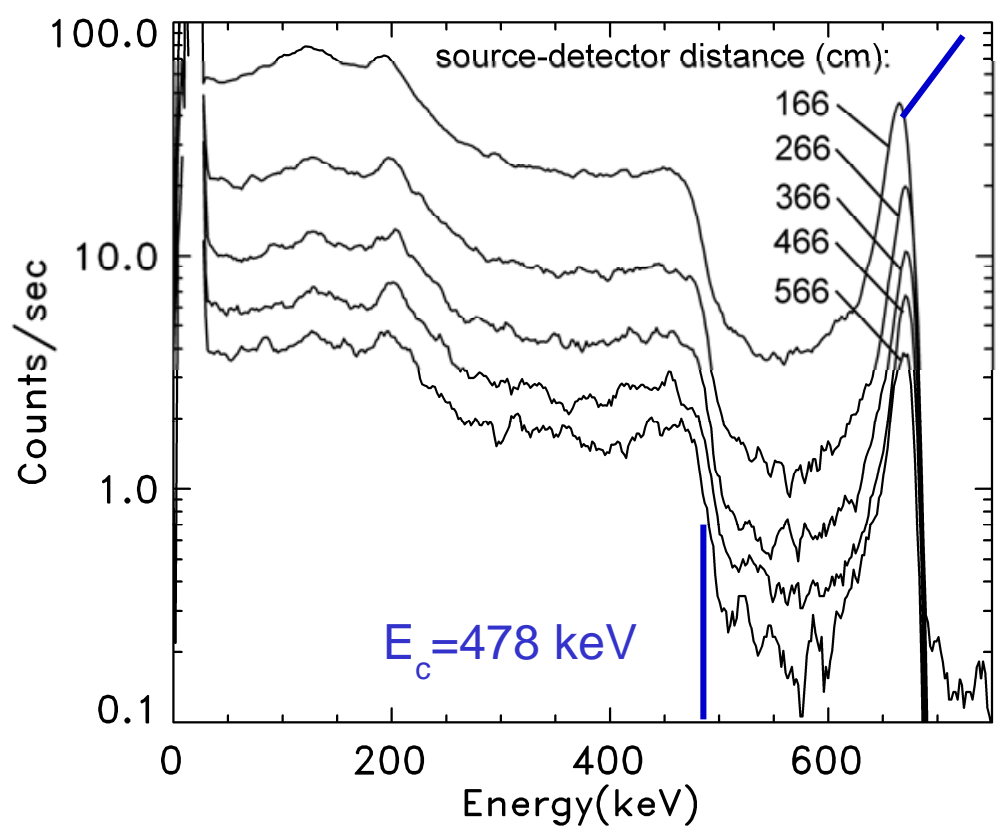

Peak

Fig. 20. The spectrum from the Cs-137 source recorded by a CdTe photon-counting detector with the indicated source to detector distances.

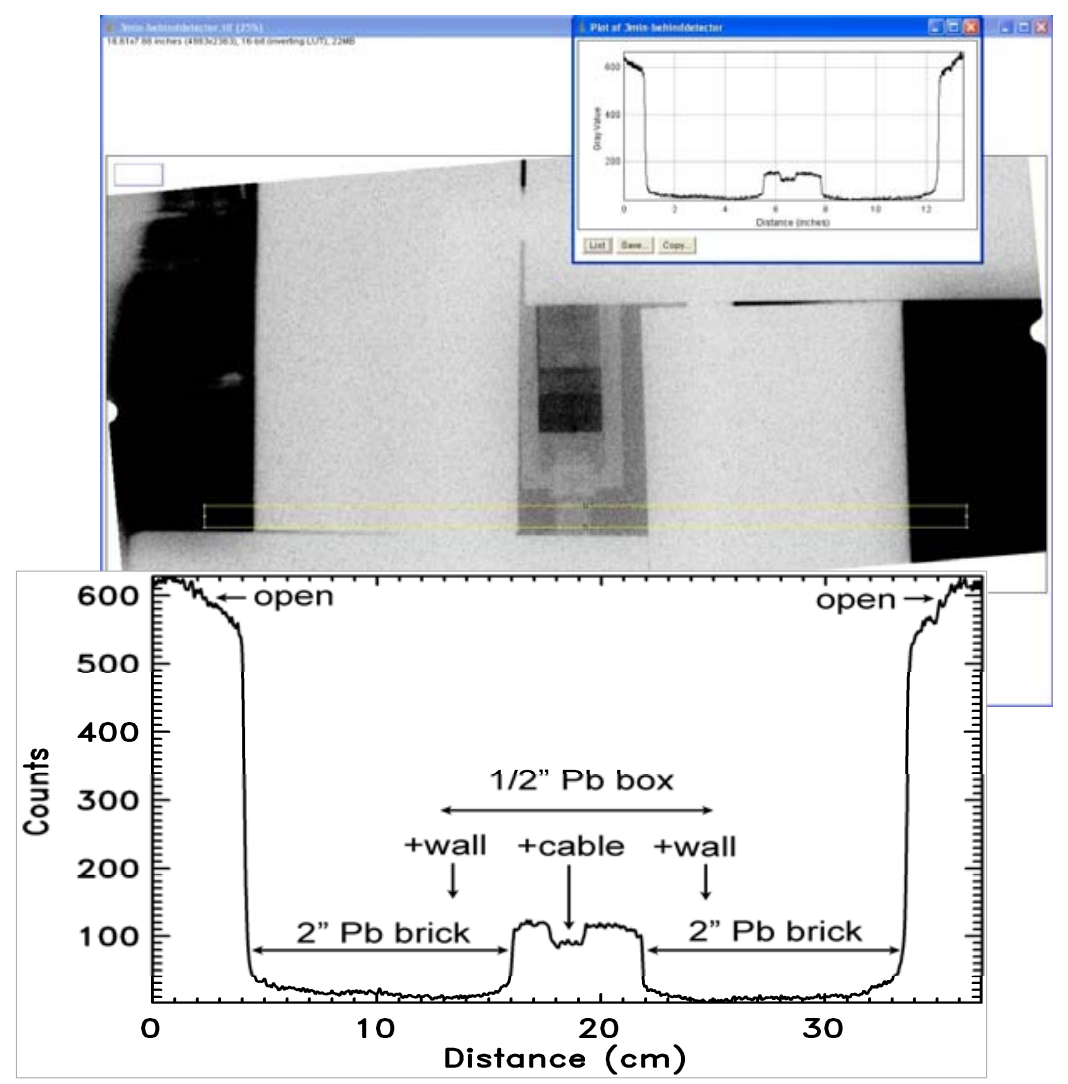

Fig. 21. Top is the $662 \mathrm{keV}$ image recorded on the Logos image plate, and bottom is a horizontal lineout through the image. 